\title{
EFICIÊNCIA TÉCNICA DA EXTRAÇÃO DO PEQUI NO CEARÁ ${ }^{1}$
}

\author{
Erivelton de Souza Nunes ${ }^{2}$ \\ Eliane Pinheiro de Sousa ${ }^{3}$ \\ Nagilane Parente Damasceno ${ }^{4}$
}

Resumo: O pequi é um fruto nativo que apresenta relevante potencial nutritivo e desempenha importante papel na geração de emprego e renda nos locais de sua ocorrência. Dessa forma, este estudo busca mensurar os escores de eficiência técnica dos extrativistas de pequi cearenses. Para cumprir o objetivo proposto, empregou-se o modelo de análise envoltória dos dados (DEA). Os dados são de natureza primária, coletados em 2013, diretamente com uma amostra de 86 extrativistas de pequi, localizados no município cearense de Crato. Os resultados indicaram que os extrativistas de pequi cearenses podem reduzir, em média, os custos com insumos em $72 \%$ e $21 \%$, respectivamente, nos modelos com retornos constantes e variáveis de escala, sem comprometer a produção. Ademais, observou-se que, sob a pressuposição do modelo com retornos constantes de escala, a maior parte dos extrativistas apresentou escores de eficiência inferiores a 0,5 , porém, quando se inclui uma restrição de convexidade, parcela majoritária dos extrativistas obtém nível ótimo de eficiência.

Palavras-chave: eficiência, pequi, Ceará.

1 Recebido em: 01/07/2014; Aceito em: 07/10/2014.

2 Graduando em Ciências Econômicas pela Universidade Regional do Cariri (URCA) e Bolsista PIBIC URCA. E-mail: erivelton.s.n@hotmail.com

3 Doutora em Economia Aplicada pela Universidade Federal de Viçosa (UFV) e Professora do Departamento de Economia da Universidade Regional do Cariri (URCA).E-mail: pinheiroeliane@hotmail.com

4 Doutoranda em Desenvolvimento e Meio Ambiente pela Universidade Federal do Ceará (UFC) e Professora do Departamento de Economia da Universidade Regional do Cariri (URCA).E-mail: nagilaneparente@yahoo. com.br 


\begin{abstract}
Pequi is a native fruit that has relevant nutritional potential and plays an important role in the generation of employment and income in the places where it occurs. Therefore, this study aims to measure the technical efficiency scores of pequi extractivists of the state of Ceará. In order to achieve the proposed goal, the data envelopment analysis model (DEA) has been employed. The data are of primary nature, collected in 2013 , directly with a sample of 86 pequi extractivists located in the municipality of Crato, in the state of Ceará. The results indicated that the pequi extractivists of the state of Ceará can reduce, on average, the costs with raw materials in $72 \%$ and $21 \%$, respectively, in the models with constant and variable returns to scale, without compromising the production. In addition, it has been observed that, about the assumption of the model with constant returns to scale, the majority of the extractivists showed efficiency scores below 0.5 , however, when one includes a convexity restriction, the majority of the extractivists obtain optimal efficiency level.
\end{abstract}

Keywords: efficiency, pequi, Ceará.

\title{
1. Introdução
}

O pequi tem grande importância no Cerrado Brasileiro, estando distribuído espacialmente entre o Centro-Oeste, Leste de Rondônia, Norte e Oeste de Minas Gerais, Sudoeste do Pará, Tocantins, extremo Sul do Piauí, Oeste da Bahia, Sul do Ceará e demais localidades. Esse fruto apresenta exploração econômica feita de modo extrativista, sendo relevante para várias famílias, na medida em que serve como alimento nutritivo e mostra ser uma boa opção de rentabilidade para os extrativistas e comerciantes. Ademais, sua árvore tem importância verificada em um conjunto de valores que englobam desde o meio ecológico até o aspecto econômico (SILVA, 2009).

De acordo com Carvalho (2009), o pequi se destaca pelas suas múltiplas utilidades. As folhas de sua árvore, assim como o próprio pequi, podem ser aproveitadas no consumo de animais bovinos, embora os espinhos presentes no fruto do pequizeiro possam trazer riscos à saúde desses animais. Como alimento, o pequi tem enorme valorização por parcela da população presente no meio rural, pois, conforme análise química, 
ele é o que apresenta maior quantidade de vitamina A, contendo outros fatores importantes para a saúde humana, como a riboflavina, fósforo, ferro e cobre.

O pequizeiro também tem relevância para a atividade apícola e para o setor energético do país, já que produz pólen e néctar, e sua madeira produz carvão de ótima qualidade. Sua madeira pode ser utilizada na indústria moveleira, sendo uma das poucas madeiras presentes no país com resistência à poluição que os detritos dos currais produzem. Ademais, também tem importância na medicina popular, haja vista que o óleo retirado do pequi tem grande quantidade de vitamina $\mathrm{A}$ e E (CARVALHO, 2009).

De modo geral, embora a exploração econômica de pequi ocorra de maneira extrativista, várias famílias têm recebido importantes benefícios no período de safra. Segundo Nunes et al. (2013), até 67,44\% dos extrativistas de pequi cearenses recebem até um salário mínimo mensal na época de safra, e grande parte desses extrativistas considera essa atividade a principal.

A atividade de extração e comercialização do pequi para a agricultura familiar tem representado uma alternativa de boa lucratividade, uma vez que esta atividade tem custos mínimos de produção. Contudo, alguns problemas como domesticação da espécie, erosão genética, complexidade das técnicas de propagação e ausência de padrões de qualidade na comercialização, dada pela exploração econômica informal de boa parte do que é produzido pelos extrativistas, são limitações que ameaçam a futura exploração sustentável do pequi (OLIVEIRA et al., 2005).

Para Santos et al. (2013), é preciso mudar a forma de exploração do pequi, apesar de notória a relevância e o grande mercado presente para sua produção e comercialização, pois Pozo (1997) já especulava sobre sua possível escassez em futuro breve. Portanto, é importante averiguar se essa atividade está sendo explorada de forma eficiente. 
Dados do IBGE (2013) mostram que, em 2011, o Brasil teve uma produção de 7.047 toneladas de amêndoas de pequi, com o valor da produção na extração vegetal (amêndoa) estimado em R \$ 11.113.000,00, tendo o estado do Ceará respondido por $60,75 \%$ dessa produção e por $46,64 \%$ desse valor, adquirindo destaque nacional em tal atividade. O município cearense de Crato foi responsável por aproximadamente $78,16 \%$ da produção estadual. Assim, o presente estudo focou este município como representante do estado do Ceará.

De posse dessas considerações, essa atividade tem despertado interesse, fazendo parte como objeto de pesquisas recentes, por exemplo, de Feitosa et al. (2005), Oliveira et al. (2005), Afonso (2008), Rocha et al. (2008), Silva (2009), Buzin et al. (2010), Sant'Anna (2011), Cândido et al. (2012), e Nunes et al. (2013). Entre esses estudos, nenhum deles se preocupou em avaliar a eficiência técnica dos extrativistas dessa atividade. Desse modo, é importante que sejam desenvolvidos estudos que contemplem essa questão.

De acordo com Gomes et al. (2005), a averiguação da eficiência de unidades produtoras exerce papel importante para fins estratégicos, permitindo a comparação de unidades produtoras para planejamento, por meio da apreciação dos resultados da utilização de combinação de fatores distintos, e para tomada de decisão, como um modo de aprimorar o desempenho efetivo.

Assim, em face da importância desempenhada pelo extrativismo e comercialização do pequi para o desenvolvimento socioeconômico do estado do Ceará, este estudo busca analisar a eficiência técnica dos extrativistas de pequi pertencentes a essa área de estudo. Especificamente, pretende-se mensurar os escores de eficiência técnica e de escala de tais extrativistas e verificar o tipo de rendimento de escala desses extrativistas. 


\section{Revisão de literatura}

\subsection{Estudos sobre a atividade extrativa do pequi em diferentes abordagens}

Conforme descrito, a atividade extrativa do pequi tem sido abordada em múltiplos aspectos e em diferentes recortes geográficos na literatura. Feitosa et al. (2005) buscaram analisar a importância econômica do pequi nas áreas de cerrados no Sudeste Paraense. Para tal, realizaram uma pesquisa de campo com os pequenos produtores dos Projetos de Assentamentos no município de Santa Maria das Barreiras, em 2004. Esses dados foram apresentados por meio de análise tabular e gráfica. Os resultados mostraram que, apesar do alto valor comercial do pequi, os pequenos produtores não dão importância para essa fruta, ficando à mercê dos comerciantes de feira, que fazem a coleta e a comercialização.

A importância socioeconômica do pequi também foi objeto de estudo de Oliveira et al. (2005) e Afonso (2008), respectivamente, na região de Iporá, no Oeste do estado de Goiás e na Cooperativa de Pequi em Japonvar, no estado de Minas Gerais. Além de tratar dessa questão, Oliveira et al. (2005) também buscaram identificar os principais agentes do arranjo extrativista e descrever a dinâmica da comercialização do pequi. Para tal, foram realizadas entrevistas semiestruturadas com os atores chave, os atuais centros de comercialização, assim como as principais regiões ou municípios fornecedores.

Verificou-se que a atividade de coleta e comercialização do pequi para a agricultura familiar apresenta boa rentabilidade, principalmente considerando que não existem custos de produção. Além do potencial como gerador de renda alternativa para a agricultura familiar, o arranjo extrativista do pequi gera emprego no meio rural e urbano. Esses resultados sobre a relevância desempenhada pelo pequi na geração de trabalho e renda são corroborados no estudo de Afonso (2008). Os métodos utilizados por esse autor foram a estatística descritiva e a análise 
de correlação e fatorial, constatando valorização do produto, redução de desperdício dos frutos, ampliação do tempo de comercialização e busca de melhorias sociais e ambientais.

Visando a uma abordagem quantitativa sobre a dinâmica espacial e temporal do extrativismo do pequi no Brasil, Rocha et al. (2008) utilizaram dados secundários obtidos no Instituto Brasileiro de Geografia e Estatística para o período de 1975 a 2006. Os resultados mostraram que a atividade extrativa de pequi no Brasil apresenta grandes oscilações de produção, tanto em relação às safras regionais ao longo do tempo quanto em termos geográficos, estando polarizada em algumas microrregiões específicas, tendo a maior expressividade sido verificada no Crato, no estado do Ceará, seguida pela microrregião de Montes Claros, no estado de Minas Gerais.

Esta última região referenciada foi objeto de estudo de Silva (2009), que buscou analisar as dinâmicas territoriais, econômicas, sociais, culturais e ambientais engendradas pelo pequi. Para alcançar esse objetivo, foram realizadas entrevistas com vendedores de pequi. Os resultados mostraram que o pequi proporciona uma das principais fontes alternativas de renda no Norte de Minas durante sua safra, mas apontam algumas dificuldades enfrentadas na cidade de Montes Claros, como a falta de espaço apropriado para a comercialização, a fiscalização urbana e a intervenção de atravessadores.

A lucratividade de um projeto de pequi é confirmada por Buzin et al. (2010) no território Kalunga, no estado de Goiás, por meio da metodologia System Dynamics, considerando um período de 20 anos de instalação de um possível empreendimento. Outra simulação do extrativismo sustentável do pequi foi adotada por Sant'Anna (2011), que buscou avaliar a viabilidade de geração de renda pelo uso sustentável da Reserva Legal no Cerrado, comparando-a com a rentabilidade obtida em outros cultivos como a soja. Para tal, utilizou simulações de Monte Carlo com base em dados secundários coletados nas Centrais Estaduais de Abastecimento (Ceasas), Companhia Nacional de Abastecimento 
(Conab) e Empresa Brasileira de Pesquisa Agropecuária (Embrapa). Os resultados constataram que a extração sustentável de pequi pode ter um uso econômico viável na Reserva Legal do Cerrado.

Cândido et al. (2012) analisaram a extração do pequi no norte de Minas Gerais utilizando a abordagem do Sistema Agroalimentar Localizado (Sial), pela organização de produtores e empresas associadas por suas características e seu funcionamento a um território específico. Foram verificadas a precariedade da integração territorial dessa região, influenciando produção, obtenção de insumos e escoamento dos produtos derivados do pequi assim como a precariedade das redes de transporte e a distância dos grandes centros consumidores.

Por sua vez, Nunes et al. (2013) buscaram mensurar um índice de desempenho competitivo dos extrativistas de pequi no Ceará. Para tal, foram utilizados os métodos de estatística descritiva e análise fatorial por meio do levantamento de dados primários com produtores de pequi residentes no município cearense de Crato. Os resultados indicaram que parcela majoritária dos extrativistas de pequi apresentou baixo desempenho competitivo.

Conforme constatado nos estudos recentes descritos nessa breve revisão de literatura, utilizando vários métodos analíticos e abordagens em diferentes áreas de estudo, o pequi reveste-se de importância. Entretanto, não se encontrou nenhum estudo que analise a eficiência técnica dessa atividade de extração vegetal utilizando o método de Análise Envoltória dos Dados. Portanto, este estudo pretende preencher essa lacuna.

\subsection{Aplicações da eficiência técnica às atividades extrativistas}

A eficiência técnica tem sido utilizada frequentemente em pesquisas relacionadas às atividades agropecuárias, que, conforme Almeida (2012), incluem dados de produção animal, produção vegetal, silvicultura e extração vegetal, fazendo o pequi parte desta última atividade. Como 
mencionado, a literatura econômica não dispõe de estudos que tratem especificamente dessa atividade de forma desagregada, embora alguns trabalhos, como os de Marinho e Carvalho (2002), Nogueira (2005) e Almeida (2012), contemplem a extração vegetal em suas análises.

Marinho e Carvalho (2002) procuraram comparar as variações da eficiência técnica na agricultura entre as regiões brasileiras, entre os anos de 1970 e 1996 . Na ocasião, o valor da produção agrícola contemplava atividades agropecuárias, horticultura, silvicultura, floricultura, avicultura, sericultura e extração vegetal. Os dados foram obtidos nos Censos Agropecuários do IBGE. Esses autores utilizaram o método de análise envoltória dos dados (DEA) e constataram que os financiamentos da produção e os investimentos em máquinas podem contribuir para a redução das ineficiências técnicas na agricultura.

Utilizando dados provenientes do Censo Agropecuário 1995/1996 e do Censo Demográfico de 2000 do IBGE, Nogueira (2005) buscou classificar a eficiência técnica e de escala do setor agropecuário das 544 microrregiões brasileiras mediante o método de Análise Envoltória dos Dados, em que a extração vegetal fez parte da análise. Além disso, procurou-se identificar os fatores que determinaram as distinções de eficiência técnica da atividade agropecuária entre todas as microrregiões averiguadas por meio do modelo Tobit. Os resultados mostraram que, na região Nordeste, a extração vegetal caracterizou-se por ser uma das atividades econômicas mais importantes nas microrregiões que apresentaram mais eficiência em relação às microrregiões ineficientes. Ademais, ao lado da produção de frutas, o extrativismo vegetal mostrouse mais importante para as microrregiões eficientes do Norte.

Almeida (2012), por sua vez, buscou verificar se existem divergências na eficiência técnica entre os estabelecimentos agropecuários de pequeno, médio e grande porte no Brasil, em 2006. Os dados foram retirados do Censo Agropecuário (IBGE) de 2006 e utilizou-se a abordagem de estimação de fronteiras de produção estocástica para medir a eficiência técnica. Esta pesquisa utilizou a extração vegetal como parte integrante 
da variável referente ao valor da produção da agropecuária. Os resultados mostraram que a eficiência técnica na agropecuária brasileira varia entre regiões geográficas e dentro delas, se diferenciando entre os estratos dos estabelecimentos.

\section{Metodologia}

\subsection{Métodos de análise e variáveis utilizadas}

A ferramenta metodológica utilizada neste trabalho foi o método de Análise Envoltória de Dados (Data Envelopment Analysis - DEA), para determinar os índices de eficiência dos extrativistas de pequi cearenses. Para Gomes e Baptista (2004), a eficiência referente às unidades produtoras pode ser analisada por métodos paramétricos, em que se estimam as fronteiras de produção por meio de ferramentas econométricas ou métodos não paramétricos, em que se aplica a programação matemática linear.

Conforme Cooper et al. (2002), a possibilidade de obtenção de relações entre múltiplos produtos e insumos de maneira menos complexa, a identificação das ineficiências existentes em cada insumo e produto e o conhecimento das unidades referenciais que podem servir como parâmetro de eficiência técnica para unidades ineficientes são as principais vantagens de aplicação deste método em relação ao emprego de outros métodos.

O método DEA, que se fundamenta nos estudos propostos por Farrell (1957) e Charnes et al. (1978), toma como base uma amostra de insumos e produtos verificados para diferentes unidades produtivas ou unidades tomadoras de decisão (DMUs - Decision Making Units), em que se pretende elaborar uma fronteira linear por partes e, mediante o emprego de medidas radiais e de função de distância, verificar a eficiência das unidades produtoras em relação à distância de fronteira construída com 
os benchmarks (os mais eficientes). Nesta pesquisa, as DMUs abordadas referem-se aos extrativistas de pequi do Ceará, representados pelo município de Crato.

Para mensurar a eficiência referente às unidades produtoras, é necessário comparar seus níveis de insumo e produtos com os níveis identificados nos benchmarks. De acordo com Charnes et al. (1994), para que uma DMU seja considerada eficiente, nenhum produto pode aumentar sua produção sem que haja acréscimo na utilização de insumo ou diminuição da produção de outro produto e/ou nenhum insumo pode ser reduzido sem ter que reduzir a produção de outro produto.

Coelli et al. (1998) sinalizam que para uma DMU ser eficiente no modelo com retornos constantes de escala, ela deverá necessariamente ser eficiente no modelo com retornos variáveis, entretanto, a recíproca não acontece. A DMU considerada terá ineficiência de escala se o escore de eficiência técnica for diferente nos dois modelos. Desse modo, Ferreira e Gomes (2009) afirmam que a eficiência técnica global das unidades produtivas pode ser dividida em dois tipos de eficiência - a pura eficiência técnica e a de escala - sendo que a última diz respeito ao quociente existente na relação entre o escore adquirido no modelo com retornos constantes de escala (CCR ou CRS) e o identificado no modelo com retornos variáveis de escala (BCC ou VRS).

O primeiro modelo foi elaborado por Charnes et al. (1978) e sugere relação proporcional entre inputs e outputs; já o segundo modelo considera a incorporação de uma restrição de convexidade ao modelo CCR, tendo sido proposto por Banker et al. (1984). Esse último modelo definido sugere a possibilidade de ocorrência de retornos variáveis de escala, que podem apresentar rendimentos crescentes ou decrescentes à escala na fronteira eficiente.

Para Coelli et al. (1998), os modelos DEA com retornos constantes e variáveis de escala podem ser apresentados, respectivamente, pelas fórmulas (1) e (2): 
$\operatorname{Min}_{\theta, \lambda} \theta$, sujeito $a:-y_{i}+Y \lambda \geq 0, \quad \theta x_{i}-X \lambda \geq 0 \quad$ e $\lambda \geq 0$

$\operatorname{Min}_{\theta, \lambda} \theta$, sujeito $a:-y_{i}+Y \lambda \geq 0, \quad \theta x_{i}-X \lambda \geq 0, \quad N_{1}{ }^{\prime} \lambda=1 \quad e \quad \lambda \geq 0$

Em que $\theta$ é o escore de eficiência de uma determinada DMU; y é o produto da DMU; $x$ é insumo; Y é a matriz de produtos (n x m); X é a matriz de insumos; $N_{1}$ é o vetor $(\mathrm{N} \times 1)$ de algarismos unitários; e $\lambda$ é o vetor de constantes que multiplica a matriz de insumos, que multiplica a matriz de insumos e produtos.

Para Ferreira e Gomes (2009), a suposição de retornos constantes de escala permite que tal tecnologia aplicada seja representada por uma isoquanta unitária. $\mathrm{O}$ escore de eficiência será igual a um sobre essa fronteira. Isso implica dizer que a DMU considerada apresenta eficiência. Um escore inferior a um, sob a ótica do insumo, sinaliza que a produção pode ser mantida com utilização de uma menor quantidade de insumos e, sob a ótica do produto, indica que se pode elevar a produção com a manutenção do nível de consumo. Neste estudo, foram mensurados os escores de eficiência para ambos os modelos usando a orientação insumo, que procura reduzir os insumos sem alterar o nível do produto.

Segundo Gomes e Baptista (2004), a existência de apenas uma observação discrepante na amostra influenciará todos os escores de eficiência, comprometendo os resultados. Assim, antes de calcular esses escores, realizou-se um teste de detecção de outliers, proposto por Sousa et al. (2005). Esses autores aplicaram uma técnica de identificação de outliers baseado no método Jackstrap, que foi elaborado com base na combinação do teste Jacknife com o método de reamostragem Bootstrap. Inicialmente, empregou-se um subconjunto de L DMUs, nomeados como bolhas, selecionado de modo aleatório. Seguindo a recomendação 
desses autores, empregou-se que as bolhas comportem 15\% da amostra de extrativistas pesquisados, que representam 13 DMUs, e a técnica do Bootstrap considerou 2.000 replicações. Em seguida, construiu-se uma medida de leverage que mede a influência de cada DMU sobre as demais, sendo que aquelas que tiverem maiores influências devem ser retiradas da análise para não comprometer as estimações do DEA. Para esses autores, o ponto de corte sugerido deve tomar como base a função Heaviside, que considera os dados obtidos dos leverages e a quantidade de DMUs K, conforme as seguintes especificações:

$$
P\left(\tilde{l_{k}}\right)=1, \text { se } \tilde{l_{k}} \leq \tilde{l} \log K \text { e } P\left(\tilde{l_{k}}\right)=0 \text {, se } \tilde{l_{k}}>\tilde{l} \log K
$$

em que $P\left(\tilde{l_{k}}\right)$ é a probabilidade da k-ésima DMU com leverage médio não ser outlier e o ponto de corte corresponde ao produto entre o leverage médio global $\tilde{l}$ e o logaritmo de K.

Ademais, utilizou-se o procedimento Bootstrap com o intuito de testar a confiabilidade dos coeficientes estimados da abordagem DEA. De acordo com Souza et al. (2011), esse método busca fazer várias reamostragens decorrentes de múltiplas interações sobre os escores de eficiência, permitindo validar ou não a média calculada por meio de intervalos de confiança obtidos.

Com relação às variáveis, são considerados dois insumos e um produto, em que os insumos correspondem aos custos com transporte e outros custos empregados na atividade extração do pequi e o produto foi representado pela quantidade produzida de pequi, sendo que as variáveis inputs foram expressas em valores reais anuais referentes a 2013, e a variável output foi expressa em termos de unidades.

\section{2. Área de estudo, fonte de dados e amostragem}

Este estudo foi feito no município de Crato, situado no sopé da Chapada do Araripe, extremo sul do Ceará, especificamente, na microrregião do Cariri. Como visto, este município foi escolhido pelo fato de parcela 
majoritária da produção de pequi no estado do Ceará ser proveniente dele. Dados do IBGE (2013) mostram que, em 2011, 78,16\% da produção de pequi no Ceará foi proveniente do município de Crato. Desse modo, nesta pesquisa, o município de Crato representou o estado do Ceará.

O estudo utiliza dados de natureza primária, coletados entre os meses de janeiro e abril de 2013, diretamente com uma amostra dos extrativistas de pequi.

Para determinação da amostra deste trabalho, aplicou-se a fórmula (4), sugerida por Fonseca e Martins (1996), empregada em populações finitas:

$$
n=\frac{Z^{2} \cdot p \cdot q \cdot N}{d^{2}(N-1)+Z^{2} \cdot p \cdot q}
$$

em que n é o tamanho da amostra; $\mathrm{z}$ é abscissa normal padronizada; $\mathrm{p}$, estimativa da proporção da característica pesquisada no universo; $\mathrm{q}=$ $1-\mathrm{p} ; \mathrm{N}$, número total de extrativistas de pequi no município cearense de Crato; e d representa o erro da amostragem.

Considerando que o número total de estabelecimentos agropecuários que exercem o extrativismo do pequi no município de Crato é de 184 , segundo dados do IBGE (2013), o erro de estimação de 5\% (d=0,05), a abscissa da normal padrão $Z=1,64$, ao nível de confiança de $95 \%$ e $p$ $=\mathrm{q}=0,5$ (na hipótese de se admitir o maior tamanho da amostra, haja vista que não se conhecem as proporções estudadas), encontrou-se um tamanho da amostra (n) igual a 125. No entanto, quando o tamanho inicial da amostra apresentar proporção igual ou acima de 5\% do total de elementos da população, a equação proposta por Pires (2006) pode ser aplicada para determinar o cálculo final do tamanho da amostra:

$$
n=\frac{n_{o}\left(1+n_{o}\right)}{N}
$$

em que no representa o tamanho da amostra definido na equação 4 . Desse modo, ao considerar a primeira amostra igual a 125 , obteve-se uma amostra definitiva de 86 questionários. 


\section{Resultados e discussão}

Antes de apresentar os resultados propriamente ditos, vale destacar que foi verificada presença de 14 outliers, que foram retirados da análise. Desta forma, considerou-se a estimação dos índices de eficiência para 72 extrativistas de pequi.

Para avaliar a eficiência desses extrativistas, foram estimados seus escores de eficiência pelo método de Análise Envoltória dos Dados sob orientação insumo. A Tabela 1 mostra os resultados das principais estatísticas descritivas das variáveis contempladas na estimação da fronteira de produção.

Tabela 1 - Estatísticas descritivas das variáveis utilizadas na mensuração dos escores de eficiência técnica dos extrativistas de pequi por meio do modelo DEA.

\begin{tabular}{lccc}
\hline Estatísticas & $\begin{array}{c}\text { Quantidade } \\
\text { produzida } \\
\text { (em unidades) }\end{array}$ & $\begin{array}{c}\text { Custos com } \\
\text { transporte } \\
\text { (em reais) }\end{array}$ & $\begin{array}{c}\text { Outros custos } \\
\text { (em reais)* }\end{array}$ \\
\hline Mínimo & 1.400 & 12,00 & 2,00 \\
Média & $29.702,78$ & 130,25 & 112,48 \\
Máximo & 208.000 & $1.152,00$ & $1.760,00$ \\
Desvio padrão & $32.312,11$ & 249,28 & 215,78 \\
$\begin{array}{l}\text { Coeficiente } \\
\text { de variação (\%) }\end{array}$ & 108,78 & 191,39 & 191,84 \\
\hline
\end{tabular}

Fonte: Elaborado pelos autores com base nos dados da pesquisa.

Nota: * Representam custos anuais referentes a lanternas, sacos de linhagem, botas, manutenção do transporte e demais custos.

Os dados indicam que os extrativistas de pequi localizados no município cearense de Crato apresentam grande heterogeneidade no que se refere à quantidade de pequi produzida, tendo sido registradas uma produção 
mínima de 1.400 unidades de pequi e uma produção máxima de 208.000 pequis. Esse resultado pode ser justificado pelo fato de alguns extrativistas se dedicarem mais à coleta do pequi, colhendo, inclusive, em matas, onde há uma grande quantidade do fruto, enquanto outros extrativistas limitam a coleta do fruto a suas propriedades e, nesse caso, a quantidade de pequizeiros geradores do fruto é significativamente menor.

No que tange aos custos da atividade de extração e comercialização do pequi no Ceará, os outros custos empregados na atividade apresentaram maior coeficiente de variação, indicando elevada disparidade entre os extrativistas entrevistados, tendo sido encontrados o maior custo no valor de $R \$ 1.760,00$ e o menor custo no valor de $R \$ 2,00$ reais, referente ao saco de linhagem reutilizável. Esse resultado corrobora o estudo de Oliveira et al. (2008), que sinalizou presença de custos mínimos nessa atividade, explicados pela prática puramente extrativista. Muitos extrativistas apresentaram custos irrisórios, que podem ser justificados em razão de muitos catadores, de maneira geral, coletarem o pequi em suas propriedades, com a utilização exclusiva de mão de obra familiar, e comercializarem a produção para atravessadores. Desta forma, incorrem em reduzidos custos com transporte, já que vendem seus frutos para atravessadores, que buscam a produção na propriedade ou em suas proximidades, sendo que o menor valor obtido foi $\mathrm{R} \$ 12,00$. Entretanto, alguns extrativistas assumem a despesa do traslado do pequi para comercialização no mercado, tendo, em média, um custo de $\mathrm{R} \$ 130,25$, ao passo que o maior custo de transporte encontrado foi de $\mathrm{R} \$ 1.152,00$.

A expressiva heterogeneidade verificada nessas variáveis utilizadas na estimação da fronteira de produção, Tabela 1, é refletida nos elevados coeficientes de variação dos escores de eficiência técnica e de escala (Tabela 2). Os dados dessa tabela revelam que o modelo com retornos constantes à escala se mostrou o mais heterogêneo, estando seu coeficiente de variação no patamar de 80,81\%. Apesar de essa medida de variabilidade ter sido menor para os escores de eficiência de escala e de eficiência técnica, admitindo a pressuposição de retornos variáveis à escala, a dispersão é considerada muito alta, já que, conforme Pimentel e 
Gomes (1985), quando os valores dos coeficientes de variação excedem $30 \%$, eles indicam variabilidade muito alta.

Na Tabela 2, verifica-se também que, em média, os escores de eficiência técnica do modelo CRS mostram que os extrativistas podem diminuir em $62 \%$ o uso de seus insumos sem comprometer a produção de pequi. Isso indica que os insumos estão sendo alocados de forma muito ineficiente. No modelo com retornos variáveis de escala, para que os extrativistas de pequi ineficientes passem a fazer parte da fronteira de retornos variáveis, é necessário que reduzam em 17\% a utilização de tais insumos. No tocante à eficiência média de escala, há a possibilidade de esses extrativistas aumentarem suas escalas de produção em $50 \%$.

Tabela 2 - Estatísticas descritivas e intervalo de confiança dos escores de eficiência técnica com retornos constantes à escala (CRS), com retornos variáveis à escala (VRS) e dos escores de eficiência de escala dos extrativistas de pequi, no Ceará, 2012.

\begin{tabular}{lccc}
\hline \multirow{2}{*}{ Estatísticas descritivas } & \multicolumn{2}{c}{ Eficiência técnica } & \multirow{2}{*}{$\begin{array}{c}\text { Eficiência de } \\
\text { escala }\end{array}$} \\
\cline { 2 - 3 } & CRS & VRS & \\
\hline Mínimo & 0,03 & 0,06 & 0,03 \\
Máximo & 1,00 & 1,00 & 1,00 \\
Desvio padrão & 0,31 & 0,31 & 0,31 \\
Coeficiente de variação (\%) & 80,81 & 38,02 & 62,77 \\
Média observada & 0,38 & 0,83 & 0,50 \\
\hline Intervalos de confiança (95\%) da média & & \\
\hline Mínimo & 0,32 & 0,76 & 0,43 \\
Máximo & 0,45 & 0,90 & 0,57 \\
\hline
\end{tabular}

Fonte: Elaborado pelos autores com base nos dados da pesquisa.

Como o modelo DEA é um método determinístico, qualquer valor diferente da unidade, que expressa a eficiência máxima, pode ser considerado ineficiente, o que gera oportunidade para classificações errôneas (SOUZA et al., 2011). Assim, para remover essa limitação, 
utilizou-se o procedimento estatístico de Bootstrap, adotado por esses autores. A Tabela 2 mostra ainda os intervalos de confiança a 95\% de probabilidade para as médias de eficiência, considerando o processo de reamostragem de 1.000 interações. Conforme se constata, admitindo um intervalo de $95 \%$ de probabilidade, os níveis de eficiência dos extrativistas de pequi no Ceará se distribuem, em média, entre $32 \%$ e $45 \%$ no modelo CRS, entre $76 \%$ e $90 \%$ no modelo VRS e entre $43 \%$ e $57 \%$ no modelo de eficiência de escala.

A Tabela 3 mostra as participações absolutas e relativas dos extrativistas de pequi nos intervalos de medidas de eficiência técnica para os modelos em que se empregam retornos constantes à escala (CRS) e retornos variáveis à escala (VRS), assim como a eficiência de escala. Como se pode verificar, quando se adota o modelo com retornos constantes à escala, a maior parte dos extrativistas de pequi entrevistados apresentou escores de eficiência abaixo de 0,5 . Por outro lado, apenas seis extrativistas mostraram ter nível máximo de eficiência, ou seja, somente $8,33 \%$ do total de extrativistas de pequi considerados no estudo mostraram empregar de maneira totalmente racional os insumos do processo produtivo, servindo como referência para os outros extrativistas cearenses no modelo CRS. Esses extrativistas de pequi totalmente eficientes foram aqueles que conseguiram utilizar uma melhor combinação entre custos e produção, haja vista terem obtido produções significativas de pequi com custos extremamente reduzidos, participado de cursos de capacitação e terem vínculos com associações. 
Tabela 3 - Distribuição absoluta e relativa dos extrativistas de pequi do Município de Crato, conforme intervalos de medidas de eficiências técnicas e de escala

\begin{tabular}{|c|c|c|c|c|c|c|}
\hline \multirow{3}{*}{$\begin{array}{l}\text { Medidas de } \\
\text { Eficiência }\end{array}$} & \multicolumn{4}{|c|}{ Eficiência Técnica } & \multirow{2}{*}{\multicolumn{2}{|c|}{ Eficiência de Escale }} \\
\hline & \multicolumn{2}{|c|}{ CRS } & \multicolumn{2}{|c|}{ VRS } & & \\
\hline & fi & $\%$ & fi & $\%$ & fi & $\%$ \\
\hline $\mathrm{E}<0,25$ & 33 & 45,83 & 8 & 11,11 & 21 & 29,17 \\
\hline $0,25 \leq \mathrm{E}<0,50$ & 16 & 22,22 & 7 & 9,72 & 16 & 22,22 \\
\hline $0,50 \leq \mathrm{E}<0,75$ & 10 & 13,89 & 3 & 4,17 & 15 & 20,83 \\
\hline $0,75 \leq \mathrm{E}<1,0$ & 7 & 9,72 & 1 & 1,39 & 14 & 19,44 \\
\hline $\mathrm{E}=1,0$ & 6 & 8,33 & 53 & 73,61 & 6 & 8,33 \\
\hline Total & 72 & 100,00 & 72 & 100,00 & 72 & 100,00 \\
\hline
\end{tabular}

Fonte: Elaborado pelos autores com base nos dados da pesquisa.

Em relação ao modelo com retornos variáveis à escala (VRS), 15 extrativistas obtiveram escores de eficiência inferiores a 0,5 , enquanto 53 tiveram nível ótimo de eficiência, perfazendo aproximadamente $73,61 \%$ da amostra pesquisada. Isso indica que 47 extrativistas de pequi a mais do que no modelo CRS estão na fronteira de retornos variáveis, porém não se encontram na fronteira de retornos constantes, sinalizando que o problema desses extrativistas não está associado à utilização excessiva de insumos, mas à escala inadequada de produção. Assim, parcela majoritária dos extrativistas apresentou escores de eficiência de escala inferiores à unidade, sendo que somente $8,33 \%$ deles, isto é, seis extrativistas de pequi, obtiveram escore máximo de eficiência de escala, não apresentando problemas quanto à escala de produção.

Como parcela majoritária $(91,67 \%)$ dos extrativistas cearenses de pequi apresenta ineficiência de escala, torna-se relevante identificar se essa ineficiência pode resultar da presença de retornos crescentes ou decrescentes à escala. A Tabela 4 mostra as participações absolutas e relativas dos tipos de retornos de escala dos extrativistas de pequi. Esses dados indicam predominância de retornos crescentes de escala, 
evidenciando que o aumento da produção de pequi ocorre em virtude dos custos médios decrescentes.

Tabela 4 - Distribuições absolutas e relativas dos extrativistas de pequi no Ceará, segundo os tipos de retornos de escala.

\begin{tabular}{lcc}
\hline Tipo de retornos de escala & fi & $\%$ \\
\hline Crescente & 48 & 66,67 \\
Constante & 6 & 8,33 \\
Decrescente & 18 & 25,00 \\
\hline Total & 72 & 100,00 \\
\hline
\end{tabular}

Fonte: Elaborado pelos autores com base nos dados da pesquisa.

Além desses resultados, verificou-se com base na pesquisa de campo que, entre os fatores produtivos considerados na análise, os outros custos concernentes aos custos com lanternas, sacos de linhagem, botas, manutenção do transporte e custos com associação registraram participação relativa de uso indevido muito maior do que os custos com transporte.

\section{Conclusões}

De acordo com os escores de eficiência técnica adquiridos pelo uso do método de análise envoltória dos dados no modelo CRS, verificou-se que os extrativistas de pequi cearenses podem diminuir, em média, a quantidade utilizada de insumos em $62 \%$, sem prejudicar a produção de pequi, e reduzir em $17 \%$ a utilização desses insumos para que os extrativistas de pequi ineficientes passem a pertencer à fronteira de retornos variáveis.

Quando se incluiu uma restrição de convexidade, 53 extrativistas de pequi, o que corresponde a 73,61\%, apresentaram escores máximos de eficiência técnica, fazendo parte da fronteira de retornos variáveis, 
no entanto, não conseguiram alcançar a fronteira de produção sob a pressuposição de retornos constantes de escala, indicando que esses extrativistas não estão utilizando insumos de modo excessivo, porém apresentaram problemas quanto à escala incorreta de produção.

Com relação à eficiência média de escala, os extrativistas de pequi cearenses podem ampliar suas escalas de produção em cerca de 50\%. Ademais, esses extrativistas mostraram resultados relevantes de heterogeneidade.

Outra inferência que pode ser retirada do estudo é que, em média, os níveis de eficiência dos extrativistas de pequi cearenses se distribuem em $38 \%$ no modelo com retornos constantes de escala, $83 \%$, pressupondo o modelo VRS, e 50\% quando se considera o modelo de eficiência de escala. Ademais, observou-se que os outros custos estão sendo alocados de forma mais ineficiente do que os custos com transporte nessa atividade.

\section{Referências}

AFONSO, S. R. Análise socioeconômica da produção de não madeireiros no Cerrado brasileiro e o caso da Cooperativa de Pequi em Japonvar, MG. Brasília: UnB, 2008. 95 p. Dissertação (Mestrado em Ciências Florestais) - Universidade de Brasília, 2008.

ALMEIDA, P. N. A. Fronteira de produção e eficiência técnica da agropecuária brasileira em 2006. Piracicaba, SP: ESALQ USP, 2012. 205 p. Tese (Doutorado em Economia Aplicada) - Escola Superior de Agricultura “Luiz de Queiroz”, Universidade de São Paulo, 2012.

BANKER, R.D., CHARNES, H., COOPER, W. W. Some models for estimating technical and scale inefficiencies in data envelopment analysis. Management Science, v. 30, n. 9, p. 1078-1092, 1984.

BUZIN, E. J. W. K.; PARREIRA, I. M.; FIGUEIREDO, R. S. Modelagem e simulação da produção de pequi no território Kalunga de Goiás, 
utilizando a metodologia System Dynamics. Enciclopédia Biosfera. Goiânia, v. 6, n. 11, p. 1-12, 2010.

CÂNDIDO, P. A.; MALAFAIA, G. C.; REZENDE, M. L. A exploração do pequi na região norte de Minas Gerais: abordagem por meio do Sistema Agroalimentar Localizado. Revista IDeAS. Rio de Janeiro, v. 5, n. 2, p. 118-138, 2012.

CARVAlHO, P. E. R. Pequizeiro Caryocar brasiliense. Comunicado Técnico 230. Colombo, PR: EMBRAPA, 2009, 10 p.

CHARNES, A., COOPER, W.W., LEWIN, A.Y., SEIFORD, L.M. Data envelopment analysis: theory, methodology, and application. Dordrecht: Kluwer Academic, 1994.

CHARNES, A., COOPER, W.W., RHODES, E. Measuring the efficiency of decision making units. European Journal of Operational Research, v. 2, n. 6, p. 429-444, 1978.

COELli, T.; RAO, D.S.P.; BATTESE, G. E. An introduction to efficiency and productivity analysis. Norwell: Kluwer Academic, 1998.

COOPER, W.W., SEIFORD, L. and TONE, K. Data Envelopment Analysis: a comprehensive text with models, applications, references and DEA-Solver Software. 3 ed. Norwell, Massachusetts: Kluwer Academic Publishers, 2002.

FARREL, M.J. The measurement of productive efficiency. Journal of the Royal Statistical Society, Series A, part III, p. 253-290, 1957.

FEITOSA, T. C.; MENEZES, A. J. E. A.; HOMMA, A. K. O.; MATOS, G. B. A importância do extrativismo do pequi na economia doméstica entre os agricultores do Sudoeste Paraense. In: Congresso da Sociedade Brasileira de Economia de Sociologia Rural, 48, 2005. Anais... Ribeirão Preto, SP: SOBER, 2005. 
FERREIRA, C. M. C.; GOMES, A. P. Introdução à Análise Envoltória de Dados: Teoria, modelos e aplicações. Viçosa, MG: UFV, 2009.

FONSECA, J. S.; MARTINS, G. A. Curso de Estatística - 6. ed. São Paulo: Atlas, 1996.

GOMES, A. P.; BAPTISTA, A. J. M. S. Análise Envoltória de Dados. In: SANTOS, M. L., VIEIRA, W.C., (ed.) Métodos Quantitativos em Economia. Viçosa, MG: UFV, 2004. p. 121-160.

IBGE-INSTITUTO BRASILEIRO DE GEOGRAFIAE ESTATÍSTICA. Produção da Extração Vegetal e da Silvicultura. Disponível em: $<$ http://www.sidra.ibge.gov.br>. Acesso em: 20 Jun. 2013.

MARINHO, E., CARVALHO, R. M. Comparações inter-regionais da produtividade Total, variação de eficiência técnica e variação tecnológica da agricultura brasileira - 1970 a 1996. In: Encontro Regional de Economia, 7, 2002. Anais. Fortaleza, CE: Banco do Nordeste, ANPEC, 2002.

NOGUEIRA, M. A. Eficiência técnica na agropecuária das microrregiões brasileiras. Viçosa: UFV, 2005. 105 p. Tese (Doutorado em Economia Aplicada) - Universidade Federal de Viçosa, 2005.

NUNES, E. S.; SOUSA, E. P.; DAMASCENO, N. P. Competitividade da extração e comercialização do pequi no Ceará. In: Congresso da Sociedade Brasileira de Economia, Administração e Sociologia Rural Nordeste, 8, 2013. Anais... Parnaíba, PI, 2013.

OLIVEIRA, E.; LONGHI, E. H.; VANDERLEI, J. C.; SILVA, I. D. C.; ROCHA, E. V. Arranjo extrativista do pequi (Caryocar brasiliense Camb.) na região de Iporá - Goiás: sustentabilidade e dinâmica da comercialização. In: Congresso da Sociedade Brasileira de Economia, Administração e Sociologia Rural, 46, 2005. Anais... Ribeirão Preto, SP: SOBER, 2005. 
PIRES, I. J. B. A pesquisa sob o enfoque da Estatística. Fortaleza: Banco do Nordeste do Brasil, 2006.

POZO, O. V. C.; BLANDÓN, M. L. Z.; GOMES, M. A. O. O pequi (Caryocar brasiliense): viabilidade socioeconômica do Cerrado no Norte de Minas Gerais. In: Congresso da Sociedade Brasileira de Economia e Sociologia Rural, 35 1997. Anais... Natal, RN: SOBER, 1997.

PIMENTEL; GOMES. Curso de Estatística Experimental. Piracicaba - SP: ESALQ/USP, 1985.

ROCHA, M. G.; ROCHA, T. C.; AGUIAR, J. L. P.; JUNQUEIRA, N. T. V. Dinâmica da produção extrativista de pequi no Brasil. In: IX Simpósio Nacional do Cerrado e II Simpósio Internacional de Savanas Tropicais, 2008. Anais... Brasília, DF, 2008.

SANT'ANNA, A. C. O uso econômico da reserva legal no Cerrado: uma simulação do extrativismo sustentável do pequi. Piracicaba, SP: USP ESALQ, 2011. 131 f. Dissertação (Mestrado em Economia Aplicada) - Universidade de São Paulo, 2011.

SILVA, M. N. S. Territorialidades do pequi: Montes Claros e o norte de Minas Gerais em questão. In: Encontro Nacional de Geografia Agrária, 19, 2009. Anais... São Paulo, SP: ENGA, 2009.

SANTOS , D.O.; MOURA, A.L.; BORGES, T.M.; FERREIRA, L.C.; NASCIMENTO, S. Qualidade microbiológica da polpa de pequi (Caryocar brasiliense Camb.) produzida no norte de Minas Gerais. Revista Brasileira de Agroecologia, v. 4, n. 2, 2009.

SANTOS, F. S.; SANTOS, R. F.; DIAS, P. P.; JUNIOR, L.A.Z.; TOMASSONI, F. A cultura do pequi (Caryocar brasiliense Camb.). Acta Iguazu. Cascavel, v.2, n.3, p. 46-57, 2013. 
SOUSA, M. C. S.; CRIBARI NETO, F.; STOSIC, B. D. Explaining DEA technical efficiency scores in an outlier corrected environment: the case of public services in Brazilian municipalities. Brazilian Review of Econometrics, v. 25, n. 2, p. 287-313, 2005.

SOUZA, U. R.; BRAGA, M. J.; FERREIRA, M. A. M. Fatores associados à eficiência técnica e de escala das cooperativas agropecuárias paranaenses. Revista de Economia e Sociologia Rural. Piracicaba, SP, v. 49, n.3, p. 573-598, 2011. 